\title{
Announcement of population data Y-chromosome STR haplotypes in the Madeira archipelago population
}

\author{
Ana Teresa Fernandes ${ }^{\mathrm{a}}$, António Brehm ${ }^{\mathrm{a},{ }^{*}, \text { Leonor Gusmão }}$, \\ António Amorim ${ }^{\mathrm{b}, \mathrm{c}}$ \\ ${ }^{a}$ Centro de Ciências Biológicas e Geológicas da Universidade da Madeira, Campus of Penteada, \\ 9000 Funchal, Portugal \\ ${ }^{\mathrm{b}}$ Instituto de Patologia e Imunologia Molecular da Universidade do Porto, Funchal, Portugal \\ ${ }^{\mathrm{c}}$ Faculdade de Ciências da Universidade do Porto, Funchal, Portugal
}

Received 6 March 2001; received in revised form 4 April 2001; accepted 6 April 2001

\begin{abstract}
Allele and haplotype frequencies of seven Y-chromosome STR loci were determined from a sample of 95 and 16 unrelated males from Madeira and Porto Santo Islands, respectively. (C) 2001 Elsevier Science Ireland Ltd. All rights reserved.

Keywords: Haplotypes; Y-chromosome; Madeira Island

Population: Total 111 healthy unrelated volunteer donors from Madeira and Porto Santo with known ancestors until at least the third generation.

DNA extraction: Chelex method.

PCR: According to DYS19, DYS389I and DYS389II, DYS390 and DYS393 [1], DYS391 and DYS392 [2].

Typing: By ABI Prism 310.

Results: Tables 1 and 2.

Analyses of data: Haplotype and gene diversity according to Nei [3].

Other remarks: No significant differences between populations of Madeira and Porto Santo Islands have been

found. A duplication of DYS391 has been detected in three individuals who to their knowledge do not share any common paternal ancestor. However, due to the rarity of the duplication and the low frequency of allele 9 , it is predictable that the three individuals share a common paternal ancestor. Haplotypes 24 and 28 are the most frequent ones (13 and 11 individuals), a pattern already found in populations from the Iberian Peninsula [4]. Interestingly, four males bearing haplotype 6 , were not previously reported in the Iberian Peninsula [4], from where supposedly the Madeirean population has come, but are found in Northern Europe. ${ }^{1}$
\end{abstract}

\footnotetext{
* Corresponding author. Tel.: +351-1291-705383; fax: +351-1291-705399.

E-mail address: brehm@uma.pt (A. Brehm).

${ }^{1}$ http://ystr.charite.de/index_mkl.html.
} 
Table 1

Allele frequencies and gene diversity value at seven Y-chromosome STR loci in the Madeira Archipelago population ${ }^{\mathrm{a}}$

\begin{tabular}{|c|c|c|c|c|c|c|c|}
\hline Allele & DYS19 & DYS389I & DYS389II & DYS390 & DYS391 & DYS392 & DYS393 \\
\hline 9 & - & - & - & - & 0.054 & - & - \\
\hline 10 & - & - & - & - & 0.514 & - & - \\
\hline $9 / 10$ & - & - & - & - & 0.027 & - & - \\
\hline 11 & - & 0.009 & - & - & 0.369 & 0.414 & - \\
\hline 12 & 0.009 & 0.180 & - & - & 0.036 & 0.036 & 0.180 \\
\hline 13 & 0.135 & 0.640 & - & - & - & 0.441 & 0.694 \\
\hline 14 & 0.613 & 0.153 & - & - & - & 0.099 & 0.117 \\
\hline 15 & 0.207 & 0.018 & 0.063 & - & - & 0.009 & 0.009 \\
\hline 16 & 0.036 & - & 0.613 & - & - & - & - \\
\hline 17 & - & - & 0.243 & - & - & - & - \\
\hline 18 & - & - & 0.072 & - & - & - & - \\
\hline 19 & - & - & 0.009 & - & - & - & - \\
\hline 20 & - & - & - & - & - & - & - \\
\hline 21 & - & - & - & - & - & - & - \\
\hline 22 & - & - & - & 0.072 & - & - & - \\
\hline 23 & - & - & - & 0.279 & - & - & - \\
\hline 24 & - & - & - & 0.550 & - & - & - \\
\hline 25 & - & - & - & 0.081 & - & - & - \\
\hline 26 & - & - & - & 0.018 & - & - & - \\
\hline $\mathrm{h}^{\mathrm{b}}$ & 0.567 & 0.539 & 0.561 & 0.613 & 0.600 & 0.628 & 0.477 \\
\hline
\end{tabular}

${ }^{\mathrm{a}} n=111$.

${ }^{\mathrm{b}} \mathrm{h}$ : gene diversity value.

Table 2

A list of 73 Y-chromosome STR haplotypes detected in 111 unrelated males in Madeira Archipelago population

\begin{tabular}{|c|c|c|c|c|c|c|c|c|}
\hline $\mathrm{H}^{\mathrm{a}}$ & DYS19 & DYS389I & DYS389II & DYS390 & DYS391 & DYS392 & DYS393 & $n^{\mathrm{b}}$ \\
\hline 1 & 12 & 13 & 16 & 24 & 11 & 14 & 13 & 1 \\
\hline 2 & 13 & 12 & 19 & 24 & 10 & 11 & 14 & 1 \\
\hline 3 & 13 & 13 & 16 & 24 & 9 & 11 & 13 & 1 \\
\hline 4 & 13 & 13 & 16 & 24 & 10 & 11 & 13 & 1 \\
\hline 5 & 13 & 13 & 16 & 24 & 11 & 11 & 13 & 1 \\
\hline 6 & 13 & 13 & 17 & 23 & 10 & 11 & 13 & 4 \\
\hline 7 & 13 & 13 & 17 & 23 & $9 / 10$ & 11 & 13 & 3 \\
\hline 8 & 13 & 14 & 15 & 24 & 9 & 11 & 13 & 1 \\
\hline 9 & 13 & 14 & 16 & 23 & 9 & 11 & 13 & 1 \\
\hline 10 & 13 & 14 & 16 & 24 & 9 & 11 & 13 & 2 \\
\hline 11 & 14 & 11 & 16 & 22 & 11 & 12 & 13 & 1 \\
\hline 12 & 14 & 12 & 15 & 24 & 10 & 13 & 13 & 1 \\
\hline 13 & 14 & 12 & 16 & 22 & 10 & 11 & 13 & 1 \\
\hline 14 & 14 & 12 & 16 & 23 & 10 & 11 & 13 & 3 \\
\hline 15 & 14 & 12 & 16 & 25 & 10 & 13 & 14 & 1 \\
\hline 16 & 14 & 12 & 17 & 24 & 11 & 14 & 12 & 1 \\
\hline 17 & 14 & 13 & 15 & 23 & 10 & 11 & 13 & 1 \\
\hline 18 & 14 & 13 & 15 & 24 & 10 & 13 & 13 & 1 \\
\hline 19 & 14 & 13 & 16 & 22 & 10 & 11 & 12 & 1 \\
\hline 20 & 14 & 13 & 16 & 23 & 10 & 13 & 13 & 1 \\
\hline 21 & 14 & 13 & 16 & 23 & 11 & 11 & 12 & 2 \\
\hline 22 & 14 & 13 & 16 & 23 & 11 & 13 & 13 & 1 \\
\hline 23 & 14 & 13 & 16 & 23 & 11 & 14 & 13 & 1 \\
\hline 24 & 14 & 13 & 16 & 24 & 10 & 13 & 13 & 13 \\
\hline 25 & 14 & 13 & 16 & 24 & 10 & 13 & 14 & 1 \\
\hline 26 & 14 & 13 & 16 & 24 & 11 & 11 & 14 & 1 \\
\hline 27 & 14 & 13 & 16 & 24 & 11 & 13 & 12 & 1 \\
\hline 28 & 14 & 13 & 16 & 24 & 11 & 13 & 13 & 11 \\
\hline 29 & 14 & 13 & 16 & 24 & 11 & 13 & 14 & 2 \\
\hline
\end{tabular}


Table 2 (Continued)

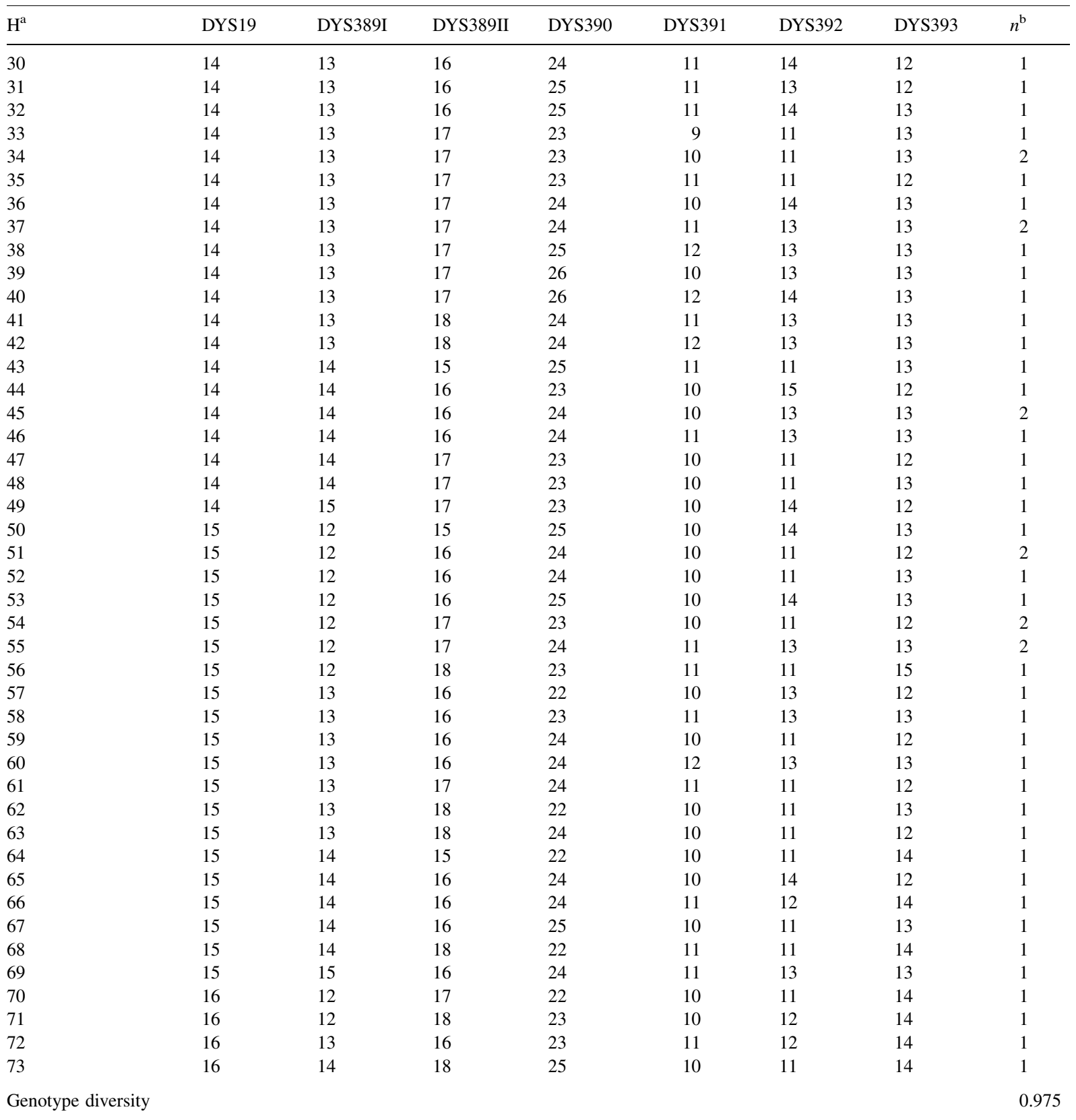

${ }^{\mathrm{a}} \mathrm{H}$ : haplotype.

${ }^{\mathrm{b}} n=$ individuals observed for each haplotype.

\section{References}

[1] L. Gusmão, A. González-Neira, C. Pestoni, M. Brión, M.V. Lareu, A. Carracedo, Robustness of the YSTRs DYS19, DYS389I and II, DYS390 and DYS393: optimisation of a PCR pentaplex, Forensic Sci. Int. 106 (1999) 163-172.

[2] M. Kayser, P. Knijff, P. Dieltjes, M. Krawczak, M. Nagy, T. Zerjal, A. Pandya, C. Tyler-Smith, L. Roewer, Applications of microsatellite-based Y-chromosome haplotyping, Electrophoresis 18 (1997) 1602-1607.

[3] M. Nei, Molecular Evolutionary Genetics, Columbia University Press, New York, 1987.

[4] A. González-Neira, L. Gusmão, M. Brión, M.V. Lareu, A. Amorim, A. Carracedo, Distribution of Y-chromosome STRdefined haplotypes in Iberia, Forensic Sci. Int. 110 (2000) 117126. 